\title{
Comparative Models for Minimizing Ergonomic Risk in Assembly Lines
}

\author{
Bautista $\mathbf{J}^{1}$, Batalla-García $\mathbf{C}^{1}$, Alfaro-Pozo $\mathbf{R}^{1}$
}

\begin{abstract}
We present a new mathematical model for the assembly line balancing problem with the objective of maximizing the line comfort to operators. Besides minimizing discrepancies between the ergonomic risks of workstations, balancing is subject to temporal and spatial conditions for the workstations. To evaluate the performance of the proposed model, we compare it with other mathematical model whose objective is minimizing the maximum ergonomic risk of a mixed-model assembly line. To compare the models, a case study linked to Nissan's engine plant in Barcelona (NMISA, Nissan Motor Ibérica - BCN).
\end{abstract}

Keywords: Assembly line; Ergonomic risk; Mathematical model; Linear programming;

\section{Introduction}

Seeing the evolutionary path from the automobile sector, we can observe how the automobile industry has had to adapt the workplace to workers' characteristics, as well as for all tools used by employees.

Various academics are agreed about defining ergonomics as the scientific discipline able to find a correct interaction between man and the different system elements that are related to environmental conditions and the workspace (Batalla, 2015).

Sometimes the sophistication of equipment and machines may hinder the adaptation of their characteristics to workers' conditions, although their high-tech pro-

\footnotetext{
${ }^{1}$ Joaquín Bautista Valhondo; Cristina Batalla García; Rocío Alfaro Pozo

( $\triangle$ e-mail:\{joaquin.bautista; rocio.alfaro; cristina.batalla\}@upc.edu)

Dpto. de Organización de Empresas. Escuela Técnica Superior de Ingeniería Industrial de

Barcelona. Universitat Politècnica de Catalunya·BarcelonaTech. Avda. Diagonal, 647.

Barcelona. This work was funded by the Ministerio de Economía y Competitividad (Spanish Government) through the FHI-SELM2 (TIN2014-57497-P) project.
} 
file. For this reason, nowadays it is important to examine and evaluate the working conditions of each workplace and then to apply ergonomic principles. In this way, the conditions, to which workers are subjected, will be improved.

Obviously, all ergonomic improvements are relevant to improve the quality, health, safety and comfort conditions of workers. Indeed, a proper workplace design aims to prevent and avoid illnesses related to inadequate working conditions, as well as ensuring more productive work.

Within the automotive industry, the mixed-model assembly lines (MMALs) are a clear example of the importance of a good workplace design. These lines are able to manufacture identical, similar or heterogeneous products and this flexibility involves variations in the consumption of components and use of resources. This, together with some technological and managerial limitations makes necessary to allocate the set of tasks needed to assembly the products, to the set of workstations of the line in the basis of some optimization criterion.

In general terms, this task assignment problem is named Assembly Line Balancing Problem (ALBP) and it has been widely discussed in the scientific literature. Indeed we can find various taxonomies in literature (Battaïa and Dolgui, 2013; Becker and Scholl, 2006).

Since the first scientific publication about this problem (Salvensson, 1955), many researches have extended the Simple Assembly Line Balancing Problem (SALBP) by means of considering additional restricted attributes. For example, Bautista and Pereira (2007) incorporated into the SALBP the linear area of workstations, defining the Time and Space Assembly Line Balancing Problems (TSALBP). On the other hand, Otto and Scholl (2011) incorporated the ergonomic risk of operations into the SALBP family. And in line with both extensions, more recently, Bautista et al. (2012) defined the TSALBP_erg family in the basis of TSALBP and limiting the minimum and maximum ergonomic risk of the line. Additionally, the authors analysed the impact of the maximum risk allowed on the number of stations of the assembly line (Bautista et al., 2013).

Latter, in a recent research, the same authors have shown how to minimize the maximum ergonomic risk of stations through Mixed Integer Linear Programming (MILP) (Bautista et al., 2015a,c) and Greedy Randomized Adaptive Search Procedures (GRASP) algorithms (Bautista et al., 2015b).

Following the works by Bautista et al., (2015a,c) and taking in mind the criteria satisfaction regarding with working conditions and production efficiency, in this paper we present a new alternative to balancing assembly line in order to maximize the comfort of operators. A comfortable assembly line implies a minimum maximum ergonomic risk and a minimum ergonomic risk range between workstations or, in other words, a minimum difference between the worst and the best workstation in regard with their ergonomic risk values.

Therefore with the aim of maximize the line comfort we present in the next section a new mathematical model to minimize the average absolute deviation of the ergonomic risk between workstations. Besides, in order to evaluate the behaviour of the new model we carry out a case study and we compare the results with 
those obtained with the model proposed by Bautista et al., $(2015 \mathrm{a}, \mathrm{c})$, that here we call it MILP-1:min-max-R and whose objective is to minimize the maximum ergonomic risk. Finally, the conclusions of this research are collected.

\section{Mathematical model}

On the basis of compatibility and adjustment functions defined by Bautista et al., (2016) and Batalla (2015), a new optimization model is proposed. The model considers the temporal, spatial and ergonomic attributes and its objective is to minimize the discrepancy between real and ideal values for the ergonomic risk of the line. In this case, the ideal value corresponds with the average risk of the line considering a number of workstations.

The parameters and variables of the model, considering only one risk factor, are the following:

Parameters

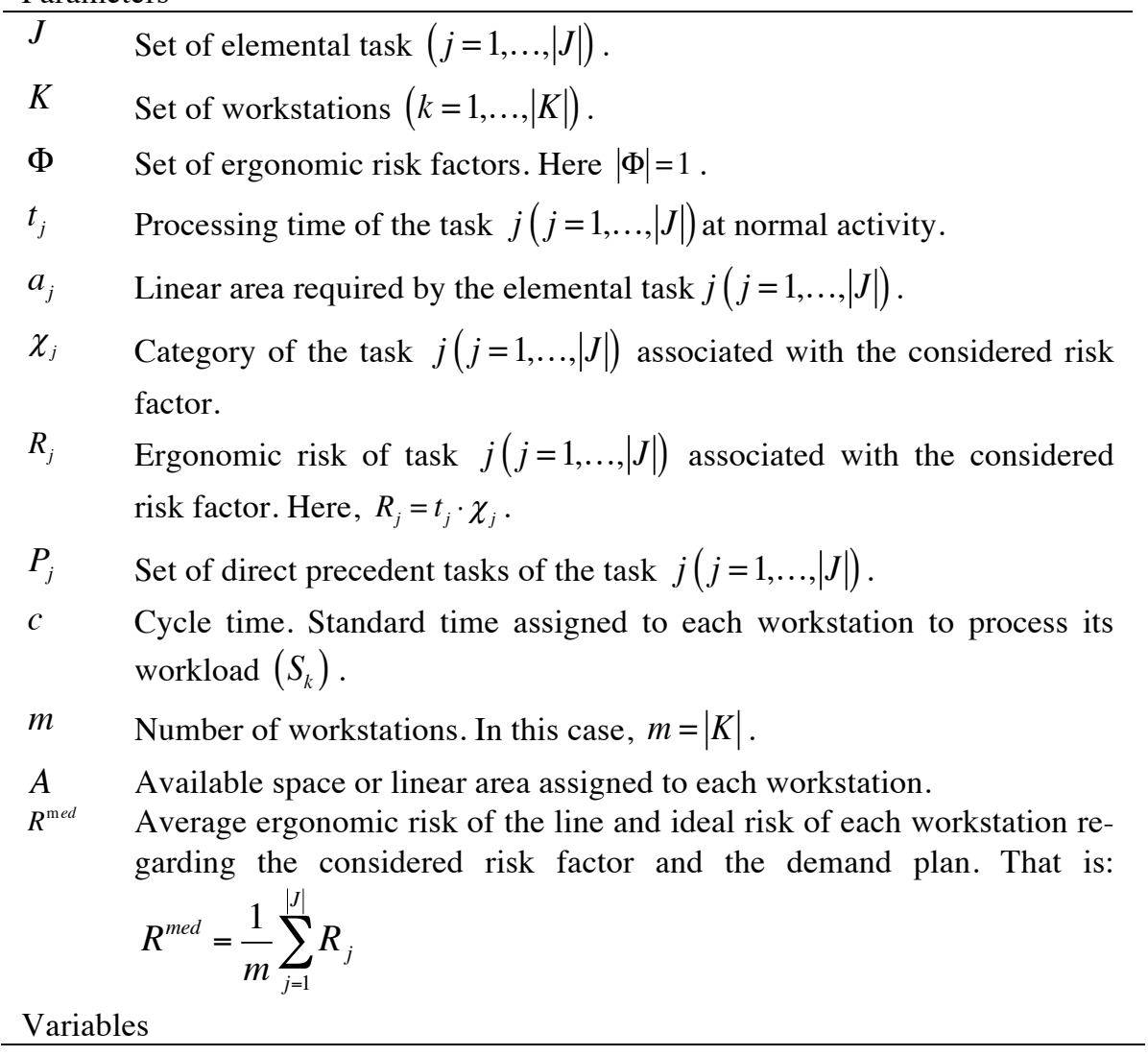




\begin{tabular}{|c|c|}
\hline$x_{j, k}$ & Binary variable equal to 1 if the elemental task $j(j=1, \ldots,|J|)$ \\
\hline & $\begin{array}{l}\text { is assigned to the workstation } k(k=1, \ldots,|K|) \text {, and to } 0 \text { other- } \\
\text { wise. }\end{array}$ \\
\hline$S_{k}$ & $\begin{array}{l}\text { Workload of workstation } K \text {. Set of tasks assigned to the } \\
\text { workstation } k \in K: S_{k}=\left\{j \in J: x_{j, k}=1\right\} \text {. }\end{array}$ \\
\hline$R\left(S_{k}\right)$ & Ergonomic risk associated with workload $S_{k}$ \\
\hline$\delta_{k}^{+}(R)$ & $\begin{array}{l}\text { Ergonomic risk excess at workstation } k \in K \text { with respect to } \\
\text { the average value } \delta_{k}^{+}(R)=\left[R\left(S_{k}\right)-R^{\text {med }}\right]^{+} .\end{array}$ \\
\hline$\delta_{k}^{-}(R)$ & $\begin{array}{l}\text { Ergonomic risk defect at workstation } k \in K \text { in regard with the } \\
\text { average value } \delta_{k}^{-}(R)=\left[R^{\text {med }}-R\left(S_{k}\right)\right]^{+}\end{array}$ \\
\hline$\Delta_{R}(R(\Phi))$ & Sum of rectangular deviations of ergonomic risk \\
\hline$A A D(R(\Phi), m)$ & $\begin{array}{l}\text { Average absolute deviations of ergonomic risk depending of } \\
\text { the number of workstations } m . A A D(R(\Phi), m)=\frac{1}{m} \Delta_{R}(R(\Phi))\end{array}$ \\
\hline
\end{tabular}

And the proposed model, named MILP-2: min_AAD-R (Average absolute deviations of ergonomic risk) is the following:

$$
\min A A D(R(\Phi))=\frac{1}{m} \Delta_{R}(R(\Phi))=\frac{1}{m} \sum_{k=1}^{|K|}\left[\delta_{k}^{+}(R)+\delta_{k}^{-}(R)\right]
$$

Subject to:

$\sum_{k=1}^{|K|} x_{j, k}=1$

$\forall j \in J$

$\sum_{j=1}^{|J|} t_{j} \cdot x_{j, k} \leq c$

$\forall k \in K$

$\sum_{j=1}^{|J|} a_{j} \cdot x_{j, k} \leq A$

$\forall k \in K$

$R\left(S_{k}\right)-\sum_{j=1}^{|f|} R_{j} \cdot x_{j, k}=0$

$\forall k \in K$

$R\left(S_{k}\right)-\delta_{k}^{+}(R)+\delta_{k}^{-}(R)=R^{\text {med }}$

$\forall k \in K$

$\sum_{k=1}^{|K|} k\left(x_{i, k}-x_{j, k}\right) \leq 0$

$\sum_{k=1}^{|K|} k \cdot x_{j, k} \leq m$

$\forall\{i, j\} \subseteq J: i \in P_{j}$

$\forall j \in J$

$\sum_{j=1}^{|J|} x_{j, k} \geq 1$

$\forall k \in K$

$R\left(S_{k}\right), \delta_{k}^{+}(R), \delta_{k}^{-}(R) \geq 0$

$\forall k \in K$

$x_{j, k} \in\{0,1\}$

$\forall j \in J \wedge \forall k \in K$

The objective function (1.1) expresses the average absolute deviation of ergonomic risk. Constraints (1.2) indicate that each task can only be assigned to one workstation. Constraints (1.3) and (1.4) impose the maximum limitation of the 
workload time and the maximum linear area allowed by workstation. Constraints (1.5) determine the real ergonomic risk associated with the workload at each workstation. Constraints (1.6) define the ergonomic risk discrepancies, both positive and negative, between the average and real values for each station. Constraints (1.7) correspond to the precedence task bindings. Constraints (1.8) and (1.9) limit the number of stations and force that there is no empty workstation, respectively. Finally, constraints (1.10) and (1.11) force the non-negativity of variables and require the assignment variables be binary.

\section{Computational Experience}

It is performed a computational experiment linked with a case study from Nissan's engine plant in Barcelona (NMISA: Nissan Motor Ibérica), in order to compare the reference model, MILP-1:min-max-R, and the model proposed here, MILP-2:min_AAD-R, that can be considered a specific case of the weighted model formulated in Bautista et al., (2015c).

Both models optimize the ergonomic risk considering a fixed number of stations and satisfying the precedence constraints, maximum cycle time and maximum available area; they differ in the optimization criterion. MILP-1 minimizes the maximum ergonomic risk of the line whereas MILP-2 minimizes the distance between the actual ergonomic risk and the desirable average value, balancing the risk as much as possible in all workstations.

For the experience we use a demand plan that consists of a daily global demand of 270 units, which are divided into 9 types of engines grouped into three families: $p_{1}, p_{2}$ and $p_{3}$ are engines for crossovers and SUVs; $p_{4}$ and $p_{5}$ are for vans; and $p_{6}, p_{7}, p_{8}$ and $p_{9}$ are intended for medium tonnage trucks.

The models was solved with the CPLEX (v11.0) software, running on a Mac Pro computer with an Intel Xeon, 3.0 GHz CPU and 2 GB RAM memory under the Windows XP operating system. In all the executions, the CPU time was limited to 2 hours for each instance due to temporal conditions imposed by the industrial environment of our case study.

The features of the computational experience are the following:

- It is selected a balanced demand plan (mix 1), whose partial demands are equal for all engine types.

- We analyse different values for the number of workstations. We run the model for the following the rank of values: $m=\{19,20,21,22,23,24,25\}$.

- The maximum cycle time is set by $c=180 \mathrm{~s}$.

- We also analyse the impact of linear area. Indeed we run the model with three values for the maximum available area; these are: $A=\{4,5, \infty\} \mathrm{m}$. 
The MILP-2 results, collected in table 1, show how the maximum ergonomic risk, which corresponds with the station with greater risk, decreases with the increase of number of workstations, for all possible evaluated area values. However, it should be noted that for maximum linear area per station of 4 meters, the model does not find line configurations with 19 and 20 workstations.

Table1 Maximum ergonomic risk of the line obtained by MILP-2: min_AAD-R.

\begin{tabular}{c|ccccccc}
\hline \multirow{2}{*}{ A } & \multicolumn{7}{|c}{$m$ (Number of workstations) } \\
& 19 & 20 & 21 & 22 & 23 & 24 & 25 \\
\hline 4 & - & - & 450 & 420 & 375 & 345 & 285 \\
5 & 440 & 390 & 320 & 300 & 275 & 265 & 255 \\
$\infty$ & 360 & 315 & 300 & 285 & 275 & 265 & 255 \\
\hline
\end{tabular}

When linear area is 4 meters, the maximum risk is 450 e-s ${ }^{2}$ and it corresponds with a line with 21 stations, whereas the minimum ergonomic risk found is $285 \mathrm{e}-\mathrm{s}$ with 25 stations. When the area is 5 meters, we have the greater maximum risk (440 e-s) with a line configuration of 19 stations and the lower maximum ergonomic risk ( 255 e-s) with 25 stations. Finally, considering boundless area, we get a line configuration with 19 stations and with the lowest maximum ergonomic risk (360 e-s), but the lower maximum ergonomic risk (that corresponds with 25 stations) is the same than the obtained with the solution with 5 meters (i.e. 255 e-s).

We can state how the area has an important effect on the line configuration. Indeed, when area is not considered $(A=\infty)$ the range of the risk, in regard with the number of stations, is 105 e-s (360-255); however, when the maximum allowable area is $A=5$ meters, the range is $185 \mathrm{e}-\mathrm{s}$. Besides, we can see that from 5 meters, the area does not affect the lower maximum ergonomic risk of the line.

Moreover, the results given by MILP-1 (Bautista et al., 2015c) are in Table 2.

Table2 Maximum ergonomic risk of the line given by MILP-1:min-max-R.

\begin{tabular}{c|ccccccc}
\hline \multirow{2}{*}{ A } & \multicolumn{7}{|c}{$m$ (Number of workstations) } \\
& 19 & 20 & 21 & 22 & 23 & 24 & 25 \\
\hline 4 & - & - & 375 & 330 & 310 & 280 & 280 \\
5 & - & - & 310 & 300 & 280 & 280 & 275 \\
$\infty$ & 350 & 315 & 300 & 285 & 275 & 270 & 255 \\
\hline
\end{tabular}

Table 2 shows the results given by MILP-1 model, that minimizes the maximum ergonomic risk, for the sweep of $m=\{19,20,21,22,23,24,25\}$ and

\footnotetext{
2 The ergo-second is the time unit, measured in seconds, used to assess the ergonomic risk of a task, with a processing time of 1 second at normal work pace, bearing a risk category of 1 . Thus, this scale measures the time spent by workers to perform a task (at normal pace) taking into account the level of the ergonomic risk to which they are exposed.
} 
$A=\{4,5, \infty\} m$. Equally to MILP-2, MILP-1 reduces the ergonomic risk while the number of workstations increases. However, MILP-1 does not find line configurations with 19 and 20 workstations when the maximum area is 4 meters and neither when the limit is 5 meters. Now, the lower maximum ergonomic risks of a line with 25 stations are 280, 275 and 255 e-s, for area limitations of 4, 5 and infinite meters, respectively. While the greater maximum ergonomic risks $(375,310)$ are obtained for lines with 21 stations in cases of 4 and 5 meters and for a line with 19 stations for $A=\infty(350 \mathrm{e}-\mathrm{s})$. In this sense, it is worth highlighting that MILP-1 obtains lower ergonomic risk than MILP-2, when the lines have smallest possible number of stations.

\section{Conclusions}

Given the importance of determining the best working conditions for workers in actual industrial environments, we have compared two mathematical models to balance assembly lines within temporal, spatial and ergonomic limitations.

The first of them, MILP-1, proposed by (Bautista et al., 2015a,c), minimizes the maximum ergonomic risk of the line, without considering the differences in risk between stations.

The second model, MILP-2, has been proposed in this paper and it is a specific case from the weighted model by (Bautista et al., 2016). This model is addressed to maximize the ergonomic comfort of the line by the minimization of average discrepancies between the actual risk of stations and the average risk resulting from the set of workstations of the line and the set of operations of the demand plan, which is the value that balances the line regarding risk.

According the results, an increase in the number of workstations leads to a reduction of the ergonomic risk of the line, whatever the model used.

Table3 Comparative of the results presented by model average absolute deviations of ergonomic risk (MILP-2) and he model of ergonomic risk minimization (MILP-1).

\begin{tabular}{c|ccccccc}
\hline \multirow{2}{*}{ A } & \multicolumn{7}{|c}{$m$ (Number of workstations) } \\
& 19 & 20 & 21 & 22 & 23 & 24 & 25 \\
\hline 4 & - & - & MILP-1 & MILP-1 & MILP-1 & MILP-1 & MILP-1 \\
5 & MILP-2 & MILP-2 & MILP-1 & IDEM & MILP-2 & MILP-2 & MILP-2 \\
$\infty$ & MILP-1 & IDEM & IDEM & IDEM & IDEM & MILP-2 & IDEM \\
\hline
\end{tabular}

Briefly, table 3 shows the winner model regardin the maximum ergonomic risk. There, we can observe how the model that minimizes the maximum ergonomic risk (MILP-1), whatever the number of stations, gives better results than the model that minimices average absolute deviations (MILP-2) when the linear area is restricted to 4 meters. When linear area is 5 meters, MILP-2 is better than MILP-1, 
except when the number of workstations is 21; however, MILP-1 does not reach solution for 19 and 20 workstations. Finally, when the area limitation does not consider, both models provide configurations with the same ergonomic risk, except when the line has 19 stations; in this case MILP-1 wins MILP-2 and 24 stations, where MILP-2 wins MILP-1.

In short, both models provide acceptable results. Even though MILP-1 wins MILP-2 in 7 times, and MILP-2 wins MILP-1 in 5 times, the results of one facing each other are rather similar. Indeed, when the best model is MILP-2 the difference with the result by MILP-1 is lower than 20 e-s.

\section{References}

Batalla C, (2015) Métodos para la incorporación del riesgo ergonómico en líneas de montaje de productos mixtos. Thesis for: Ph.D in Industrial Engineering - Universitat Politècnica de Catalunya BarcelonaTech, https://www .researchgate.net/publication/280235683

Battaïa O, Dolgui A. (2013) A taxonomy of line balancing problems and their solution approaches. Int J Prod Econ 142(2): 259-277.

Bautista J, Batalla C, Alfaro R (2015b) Algoritmos GRASP para el equilibrado de líneas con riesgo ergonómico mínimo, X Congreso Nacional sobre Metaheurísticas, Algoritmos Evolutivos y Bioinspirados, MAEB2015, https://doi.org/10.13140/2.1.4011.6005

Bautista J, Batalla C, Alfaro R (2015c) Delimiting the linear area on the problems of assembly line balancing with minimal ergonomic risk. XXI International Conference on Industrial Engineering and Operations Management 9th International Conference on Industrial Engineering and Industrial Management International IIE Conference 2015. CIO2015. https://doi.org/10.13140/RG.2.1.1543.0248

Bautista J, Batalla C, Alfaro R (2012) Incorporating Ergonomics Factors into the TSALBP, Proceedings (CD), International Conference on Advances in Production Management Systems (APMS 2012) Rodas (Greece).

Bautista J, Batalla C, Alfaro R, Cano A (2013) Impact of Ergonomic Risk Reduction in the TSALBP-1. 7th International Conference on Industrial Engineering and Industrial Management - XVII Congreso de Ingeniería de Organización (CIO 2013), Valladolid. DOI: 10.13140/2.1.2687.9041

Bautista J, Batalla-García C, Alfaro-Pozo R (2015a) Ergonomic Risk Minimisation in Assembly Line Balancing. In: Cortés, P., Maeso-González, E., Escudero-Santana A. (eds.) Ergonomic Risk Minimisation in Assembly Line Balancing. LNMIE, pp. 85-93. Springer International Publishing (2015).

Bautista J, Batalla-García C, Alfaro-Pozo R (2016) Models for assembly line balancing by temporal, spatial and ergonomic risk attributes. European Journal of Operational Research 251(3), 814-829, https://doi.org/10.1016/j.ejor.2015.12.042

Bautista J, Pereira J (2007) Ant algorithms for a time and space constrained assembly line balancing problema. Eur J Oper Res 177(3):2016-2032.

Becker C, Scholl A. (2006). A survey on problems and methods in generalized assembly line balancing. Eur J Oper Res 168(3): 694-715.

Otto A, Scholl A. (2011). Incorporating ergonomic risks into assembly line balancing. Eur J Oper Res 212(2), 277-286.

Salveson ME (1955) The assembly line balancing problema. J Ind Engineering 6(3):18-25. 